\title{
Evaluation of Functional Characteristics of 4 Oscillatory Positive Pressure Devices in a Simulated Cystic Fibrosis Model
}

\author{
Hillary Van Fleet MSc RRT, Diane K Dunn RRT-NPS, Neil L McNinch MSc RN, and \\ Teresa A Volsko MBA MHHS RRT CMTE FAARC
}

\begin{abstract}
BACKGROUND: Oscillatory positive expiratory pressure (OPEP) is an airway clearance therapy that delivers positive pressure and air-flow oscillations during exhalation. This study described functional characteristic differences of 4 OPEP devices during an active exhalation in a simulated model. We hypothesized peak pressure (Ppeak), positive expiratory pressure (PEP), oscillatory frequency (f), and pressure amplitude will differ, depending upon the device used, device resistance setting, and time (repeated consecutive active exhalations through the device). METHODS: The ASL 5000 was scripted to simulate pulmonary mechanics of a pediatric cystic fibrosis patient with moderate to severe lung disease. Airway resistance was standardized at $17.1 \mathrm{~cm} \mathrm{H}_{2} \mathrm{O} / \mathrm{L} / \mathrm{s}$, pulmonary compliance at $42.1 \mathrm{~mL} / \mathrm{cm} \mathrm{H}_{2} \mathrm{O}$, active exhalation at 22 breaths/min, and tidal volume at $409 \mathrm{~mL}$. Resistance settings for the Acapella, RC-Cornet, Flutter, and Aerobika were adjusted to low, medium, and high. Values for $\mathrm{f}$, Ppeak, PEP, and pressure amplitude were recorded for 1 min and graphically displayed. RESULTS: Significant effects for time, device, and resistance $(P<.01)$ were noted for Ppeak, PEP, and pressure amplitude at each resistance level, demonstrating that the devices functioned differently as more than one repetition of a series of consecutive active exhalations are performed. Significant interaction effects for device, resistance level, and time indicate inconsistent output for Ppeak $(P<.01)$, PEP $(P<.01)$, and pressure amplitude $(P<.01)$. Oscillatory $f$ values fell within the respective manufacturers' operational parameters. The Aerobika provided the most consistent pressure amplitude across resistance settings and produced the highest mean pressure amplitude at medium and high resistance settings. CONCLUSIONS: Statistically significant and clinically relevant variations in Ppeak, PEP, and pressure amplitude occurred between devices and within a device, as the resistance setting changed. The combination of device, time, and resistance settings affects OPEP device output for pressure, amplitude, and oscillatory frequency. Functional variations may impact therapeutic effectiveness, warranting additional study to determine clinical impact. Key words: OPEP, airway clearance, simulated model [Respir Care 2017;62(4):451-458. () 2017 Daedalus Enterprises]
\end{abstract}

\section{Introduction}

Oscillatory positive expiratory pressure (OPEP) is a variation of positive expiratory pressure (PEP). It was first

\footnotetext{
Ms Van Fleet is affiliated with the Division of Respiratory Care, Rush University College of Health Sciences, Chicago, Illinois and the Department of Respiratory Care, Dayton Children's Hospital, Dayton, Ohio. Ms Dunn is affiliated with the Department of Respiratory Care, Mr McNinch is affiliated with the Rebecca D Considine Research Institute, and Ms Volsko is affiliated with the Nursing Administration, Akron Children's Hospital, Akron, Ohio.
}

The authors have disclosed no conflicts of interest. described in the late 1990s as a secretion clearance alternative to postural drainage and percussion and/or breathing techniques. ${ }^{1,2}$ OPEP devices produce positive expiratory pressure with the addition of oscillations as the patient inspires a slightly larger than normal tidal volume and actively exhales through the device. An active exhalation

\footnotetext{
Correspondence: Teresa A Volsko MBA MHHS RRT CMTE FAARC, Akron Children's Hospital, One Perkins Square, Akron, OH 44308. E-mail: tvolsko@chmca.org.
}

DOI: $10.4187 /$ respcare. 04570 
maneuver involves a purposeful, but not forceful, movement of gas out of the lungs through the device, which lasts for $\sim 4 \mathrm{~s}$. Depending on the device, different mechanisms are used to create air-flow oscillations. The literature reports that air-flow oscillations help to mobilize secretions by reducing the viscosity and creating small bursts of air that move secretions cephalad. ${ }^{3}$ The PEP also increases functional residual capacity, augmenting collateral ventilation through the canals of Lambert and pores of Kohn. ${ }^{4}$ The OPEP maneuver is frequently followed by huff coughing, which aids in expectoration. ${ }^{5}$

There are many different types of commercially available OPEP devices. We conducted a laboratory evaluation of 4 types of airway clearance devices that combine highfrequency air-flow oscillations with PEP, specifically the Flutter (Scandipharm, Birmingham, Alabama), RC-Cornet (R Cegla GmbH \& Co, Montabaur, Germany), Aerobika (Monaghan Medical, Plattsburgh, New York), and Acapella green and Acapella blue (Smith Medical, Dublin, Ohio). Each type of device uses unique mechanisms to produce air-flow oscillations (Fig. 1).

There are 4 variables of interest with respect to the generation of air-flow oscillations with OPEP devices. Each oscillation has a peak pressure (Ppeak) and baseline PEP. The pressure amplitude is the difference between Ppeak and PEP, which is a measure of the burst of flow the device creates. The frequency is the number of air-flow oscillations occurring in a 1-min time period.

The purpose of this research project was to describe differences in functional characteristics of 4 types of commercially available OPEP devices during an active exhalation produced by a lung simulator programmed to model the pulmonary mechanics of a pediatric cystic fibrosis patient with moderate to severe lung disease. We hypothesized that Ppeak, PEP, oscillatory frequency (f), and pressure amplitude will differ significantly, depending upon the device used, resistance setting on the device, and time (repeated consecutive active exhalations through the device).

\section{Methods}

\section{Simulated Lung Model}

The ASL 5000 (IngMar Medical, Pittsburgh, Pennsylvania) was programmed to simulate the pulmonary mechanics of a pediatric patient with moderate to severe cystic fibrosis. ${ }^{6}$ Airway resistance was set at $17.1 \mathrm{~cm} \mathrm{H}_{2} \mathrm{O} / \mathrm{L} / \mathrm{s}$ and lung compliance at $42.1 \mathrm{~mL} / \mathrm{cm} \mathrm{H}_{2} \mathrm{O}$. The simulator was programed to deliver 22 breaths/min. Inspiratory time was set at $0.6 \mathrm{~s}$ and expiratory time at $2.1 \mathrm{~s}$. Each breath was programmed to deliver an active exhalation by adjusting the exhalation percentage increase and percentage release settings on the model (Fig. 2). Muscle pressure was adjusted to achieve a target tidal volume of $409 \mathrm{~mL}$.

\section{QUICK LOOK}

\section{Current knowledge}

The size of air-flow oscillations is determined by the pressure amplitude or difference in peak and expiratory pressure of an oscillation. Devices currently available on the market use a variety of mechanisms to create air-flow oscillations, which may impact the size and frequency or number of air-flow oscillations generated during a breath. Variations in functional characteristics may make device selection difficult and have the potential to impact therapeutic efficacy.

\section{What this paper contributes to our knowledge}

There were clinically relevant variations in Ppeak, PEP, and pressure amplitude between devices and for a given device, with a change in resistance settings. Individual device performance is impacted by time and resistance settings. The pressure, amplitude and oscillatory frequency differences could have an impact on patient response.

Each breath sequence was composed of a 1-min period containing 22 breaths.

\section{OPEP Device Preparation and Simulator Interface}

Before the initiation of this device evaluation, the ASL 5000 performance was verified as functional within manufacturer specifications. Each OPEP device was tested independently, with the resistance settings adjusted to produce low, medium, and high mean expiratory pressure. The resistance settings for the Acapella green, Acapella blue, Aerobika, and RC-Cornet were adjusted using a dial on the device as described in Table 1. The Flutter was adjusted by altering the position or angle (Table 1).

The devices were attached to the ASL 5000, and positioning or dial adjustments were made before obtaining data. Flutter angle was achieved by attaching this device to the lung simulator with a silicon adapter. The position of the device was then verified with an electronic level and protractor (Fig. 3). The Acapella, RC-Cornet, and Aerobika devices did not require additional adaptors; removal of the mouthpiece allowed each device to attach directly onto the ASL 5000 (Fig. 3).

\section{Data Collection}

Data were collected on each device after a 1-min stabilization period. The values for oscillatory $f$, Ppeak, PEP, and pressure amplitude were recorded by the ASL 5000 

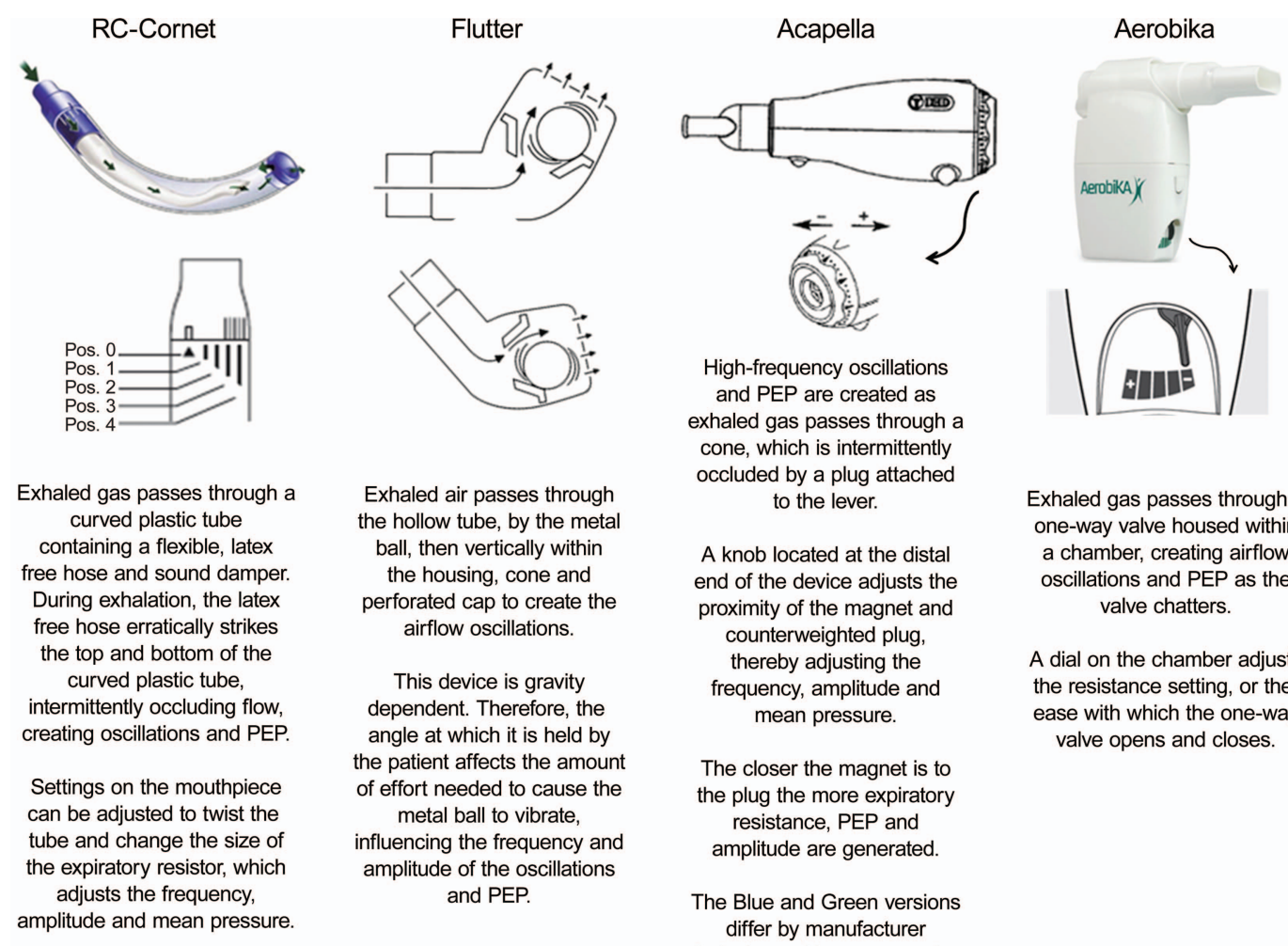
Exhaled air passes through the hollow tube, by the metal ball, then vertically within the housing, cone and perforated cap to create the airflow oscillations.

This device is gravity dependent. Therefore, the angle at which it is held by the patient affects the amount of effort needed to cause the metal ball to vibrate,

influencing the frequency and amplitude of the oscillations and PEP.

Exhaled gas passes through a one-way valve housed within a chamber, creating airflow oscillations and PEP as the valve chatters.

A dial on the chamber adjusts the resistance setting, or the ease with which the one-way valve opens and closes.

Fig. 1. Illustration of the oscillatory positive expiratory pressure (OPEP) devices tested and descriptions of their theory of operation.

software over a 1-min period. Each iteration of the experimental condition represented a unique combination of device and resistance level, which was repeated 4 times (4 breath sequences).

Values for Ppeak, PEP, and pressure amplitude were captured over 10 successive time points within each breath sequence (ie, first 10 oscillations of breath 5). These 10 repeated measures are referred to as "time" in the analysis. Oscillatory $\mathrm{f}$ and the pressure waveform were counted over a 1-s period and graphically displayed. Data were exported from the ASL5000 into Excel (Microsoft Corp, Redmond, California).

\section{Statistical Methods}

Repeated measures analysis of variance with effects for device, resistance level, breath sequence, and time along with their interactions was used to investigate variation in Ppeak, PEP, and pressure amplitude. Due to insignificant effects for breath sequence and the presence of significant interaction effects (device $\times$ resistance level $\times$ time), analyses were performed separately by resistance level using repeated measures analysis of variance with effects for device and time along with their interactions. Statistical analyses were completed using SAS 9.4/13.2 (SAS Institute, Cary, North Carolina). Unless otherwise noted, all testing was 2-tailed and evaluated at the type-1 error rate of $\alpha=.05$ level of statistical significance.

\section{Results}

\section{Simulation Model}

Repeated measures analysis of variance results for Ppeak, PEP, and pressure amplitude indicate that the effect of breath sequence was not significant, individually or as an interaction $(P$ values $>.05)$ and as such was removed from the remaining analysis. However, these results indicate that breaths delivered by the ASL 5000 with each of the 4 repeated breath sequences did not vary, providing evidence for the test-retest reliability of the experimental design. These results suggest that any differences in the variables of interest occurring during hypothesis testing were not influenced by measures across breath sequence.

\section{Hypothesis Testing}

Interaction effects for time by device by resistance setting were significant for Ppeak $(P<.01)$, PEP $(P<.01)$, and pressure amplitude $(P<.01)$, which drove the remainder of the analysis. Significant main effects for time, 


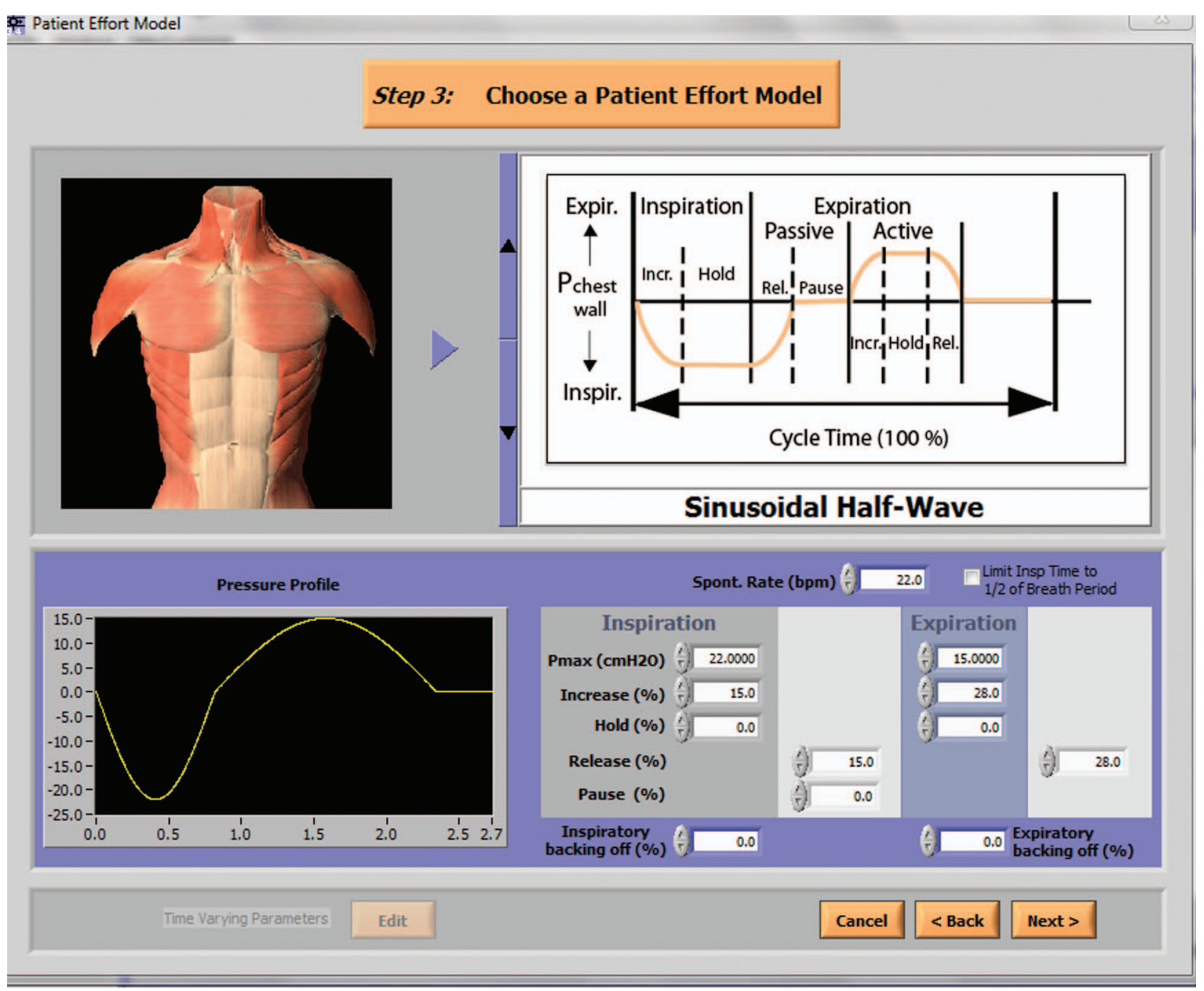

Fig. 2. Screen capture of the parameter settings used to produce active exhalation. The setting for percentage increase and percentage release settings were adjusted until a sinusoidal half-waveform with a higher, but not forceful, expiratory flow was achieved. The illustration in the top right-hand corner of the screen capture denotes that the previously described settings resulted in an active exhalation. The actual inspiratory and expiratory waveform scripted for this model is depicted in the bottom left-hand portion of the screen capture under the heading "Pressure Profile." The total cycle time of $2.7 \mathrm{~s}$ is displayed on the $\mathrm{x}$ axis. Inspiration is $0.6 \mathrm{~s}$, and expiration is $2.1 \mathrm{~s}$.

Table 1. Oscillatory Positive Expiratory Pressure Device Settings

\begin{tabular}{lccc}
\hline \hline \multirow{2}{*}{ Device } & \multicolumn{3}{c}{ Resistance Setting } \\
\cline { 2 - 4 } & Low & Medium & High \\
\hline Flutter & $0^{\circ}$ & $20^{\circ}$ & $40^{\circ}$ \\
Acapella (green/blue) & $(-)$ & Middle & $(+)$ \\
RC-Cornet & 0 & 3 & 4 \\
Aerobika & $(-)$ & Middle & $(+)$ \\
\hline
\end{tabular}

device, and resistance $(P$ value $<0.01)$ were noted for the variables of interest (Ppeak, PEP, and pressure amplitude) at each resistance level. Clinically, these statistical differences indicate real-time functional characteristic differences of the devices under similar conditions, dependent

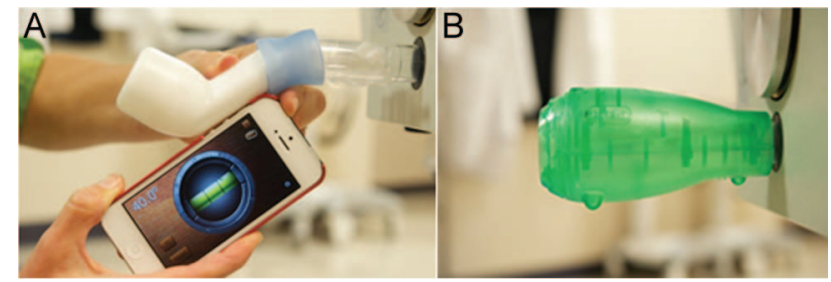

Fig. 3. Two examples of the experimental setup. A: Verification of the Flutter angle, once connected to the lung simulator. B: Connection of the Acapella to the lung simulator.

upon device and resistance setting. The significant time effects for Ppeak, PEP, and pressure amplitude demonstrate the functional characteristics of these devices, mimicking clinical use, since more than one repetition of a 
Table 2. Results of Repeated Measures Factorial Analysis of Variance for the Effect of Time, Device, Resistance Level, and Breath Sequence, Along With Interactions

\begin{tabular}{lcc}
\hline \hline Variable of Interest and Effect & $\mathrm{f}$ & $P$ \\
\hline Ppeak & & \\
Time & 66.3 & $<.001$ \\
Time $\times$ device & 5.5 & $<.001$ \\
Time $\times$ resistance level & 6.4 & $<.01$ \\
Time $\times$ breath sequence & 1.1 & .41 \\
Time $\times$ device $\times$ resistance & 3.6 & $<.001$ \\
Time $\times$ resistance $\times$ breath & 1.2 & .28 \\
Time $\times$ device $\times$ breath & 1.4 & .09 \\
PEP & & \\
Time & 12.8 & $<.01$ \\
Time $\times$ device & 6.3 & $<.001$ \\
Time $\times$ resistance level & 8.1 & $<.001$ \\
Time $\times$ breath sequence & 2 & .09 \\
Time $\times$ device $\times$ resistance & 3.2 & $<.01$ \\
Time $\times$ resistance $\times$ breath & 1.4 & .15 \\
Time $\times$ device $\times$ breath & 1.3 & .14 \\
Pressure amplitude & & \\
Time & 60.6 & $<.001$ \\
Time $\times$ device & 2.9 & $<.01$ \\
Time $\times$ resistance level & 9.1 & $<.001$ \\
Time $\times$ breath sequence & 1.6 & .16 \\
Time $\times$ device $\times$ resistance & 3.1 & .01 \\
Time $\times$ resistance $\times$ breath & 0.9 & \\
Time $\times$ device $\times$ breath & 1.3 & \\
\hline Ppeak $=$ peak pressure & & \\
PEP $=$ peak expiratory pressure & & \\
\hline & & \\
& & \\
& & \\
& &
\end{tabular}

Table 3. Count of Oscillatory Frequency in the First Breath Sequence for Each Device Across All Resistance Settings

\begin{tabular}{lccccc}
\hline \hline \multirow{2}{*}{$\begin{array}{c}\text { Resistance } \\
\text { Setting }\end{array}$} & RC-Cornet & Aerobika & $\begin{array}{c}\text { Acapella } \\
\text { Blue }\end{array}$ & $\begin{array}{c}\text { Acapella } \\
\text { Green }\end{array}$ & Flutter \\
\cline { 2 - 6 } & 12 & 12 & 9 & 15 & 15 \\
High & 14 & 10 & 15 & 15 & 15 \\
Medium & 17 & 10 & 15 & 14 & 15 \\
Low & 17 & & & & \\
\hline
\end{tabular}

series of consecutive active exhalations are performed through the device during therapy (Table 2).

The manual count of air-flow oscillations, performed on the first breath of the initial breath sequence is found in Table 3 . The oscillatory $f$ each device produced fell within the operational parameters reported by the respective manufacturer.

\section{Discussion}

Simulation studies are often used to examine device performance using models of the respiratory system. ${ }^{7,8} \mathrm{Sim}-$ ulated models carry no risk of harm and are less cumbersome to work with compared with using human or animal respiratory systems in the device evaluations. However, if the simulated model is unable to consistently replicate the model characteristics, it is difficult to distinguish whether the differences in the variable of interest were due to inconsistencies in the model or differences in the operational characteristics of the devices evaluated. Time was an important factor to consider in data analysis and enabled the researchers to determine whether the ASL 5000 was able to produce consistent breath characteristics over repeated iterations of the experiment. Repeated measures analysis of variance results for Ppeak $(P=.41)$, PEP $(P=.09)$, and pressure amplitude $(P=.61)$ for each breath sequence were nonsignificant. These results demonstrated that the breath characteristics of the cystic fibrosis model did not vary over repeated iterations of the experiment. Therefore, the differences observed in measurements are presumed to be related only to functional differences among the devices studied.

The use of the ASL 5000 enabled the simulated model to reproduce the inspiratory and expiratory maneuver required for proper use of the devices tested. However, in the model used in this device evaluation, active exhalation did not last $\geq 4 \mathrm{~s}$. The breathing frequency chosen for the model had a total cycle time of $2.7 \mathrm{~s}$. Although a 4-s exhalation is recommended by the manufacturer, children and patients with breathing frequencies $>15$ breaths/min may not be able to execute the OPEP maneuver as recommended. Our model was purposefully constructed with a breathing frequency of $>15$ breaths/min to more closely resemble the breathing frequency of patients with cystic fibrosis using OPEP in our practice.

During an OPEP maneuver, the patient supplies the flow, which is periodically occluded (partially or completely) during exhalation until the flow decays to zero at end exhalation. Energy from expiratory flow is transformed to stagnation pressure causing pressure generated by the device to rise and fall concomitantly with expiratory flow. Specifically, as expiratory flow increases and peaks the pressure generated through the device will increase and peak. Similarly, pressure decreases as expiratory flow decays, near the end of the expiratory phase. ${ }^{9}$

Figure 4 graphically displays the mean values for Ppeak, PEP, and pressure amplitude, calculated at each of the 10 time points, and illustrates the significant interaction effects by device and set resistance level. It is important to note that these mean values were calculated for graphical display purposes only, based upon breath sequence exhibiting a lack of significance; statistical analyses utilized complete data.

Flutter produced the lowest Ppeak across the range of resistance settings. The RC-Cornet produced the highest Ppeak at the high and low resistance setting. At the me- 

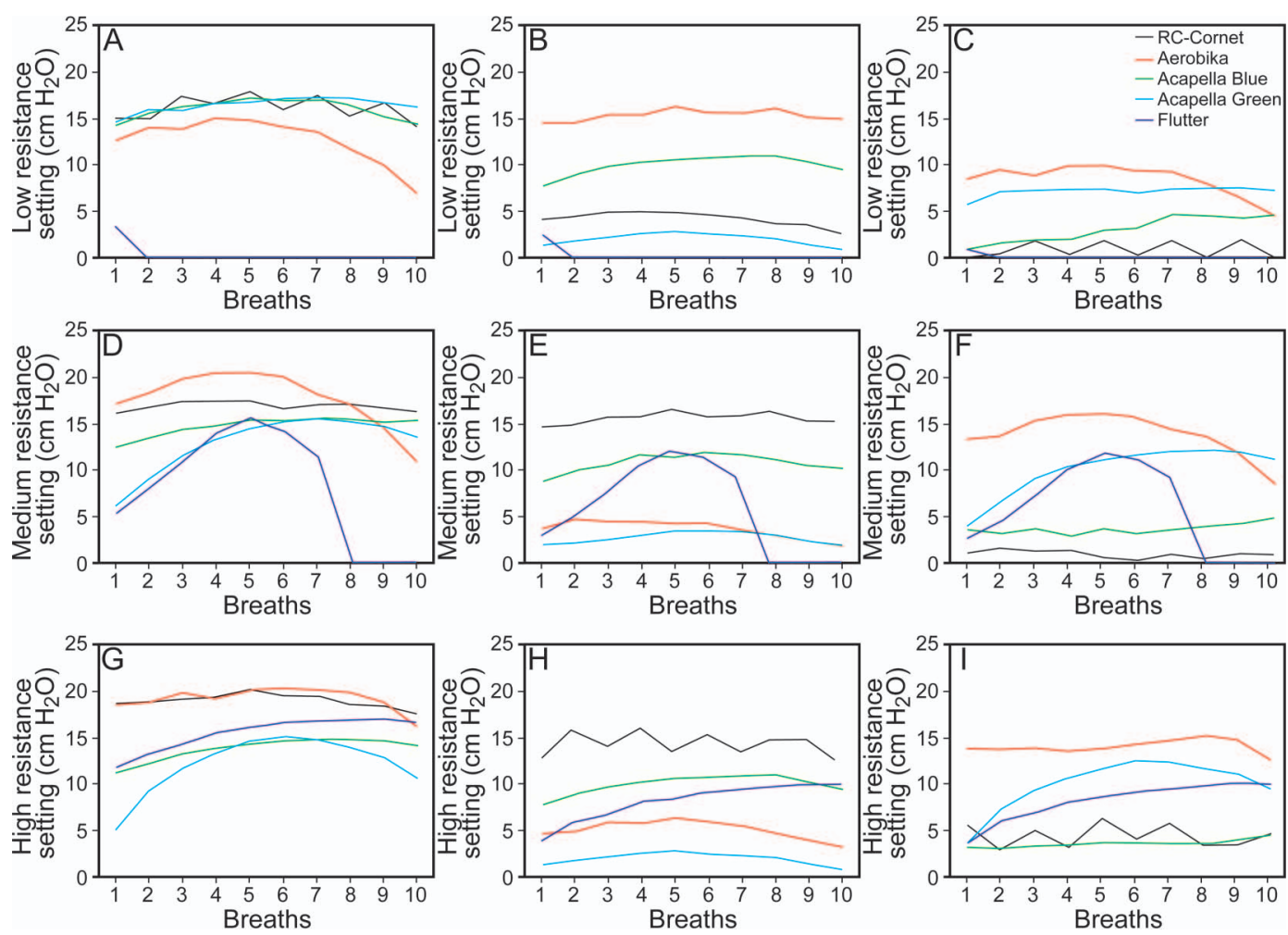

Fig. 4. Graphical display of mean values for peak pressure (A, D, G), positive expiratory pressure (B, E, H), and amplitude (C, F, I) for each device tested across the range of resistance settings.

dium resistance setting, the RC-Cornet and Aerobika produced similar Ppeak.

The lowest PEP was produced by the Acapella blue at the high resistance setting and by the Flutter at the medium and low resistance settings. The RC-Cornet produced the highest PEP across all set resistance levels and was the most markedly different from other devices tested.

A different device produced the lowest pressure amplitude at each resistance level. For example, the pressure amplitude was lowest for the Flutter and Acapella blue at the low resistance setting, for the RC-Cornet at the medium resistance setting, and for the Flutter at the high resistance setting (Fig. 4). The highest pressure amplitude was produced by the Acapella blue at the high resistance setting, the Aerobika at the medium resistance setting, and the Acapella green at the low resistance setting.

Figure 5 displays representative waveforms from the ASL 5000 output screen for each device at each resistance setting. At the lowest resistance setting, the Flutter did not produce distinguishable air-flow oscillations. These results demonstrate that there is inconsistency in the inconsistencies within and between devices. These results did not demonstrate a linear, progressive, or predictable trend. During this experiment, much like device use clinically, there was no method to detect or predict the resistance setting that would consistently produce air-flow oscillations that would produce a pressure amplitude that would be therapeutically effective.

At all resistance levels (Fig. 5), the RC-Cornet produced asymmetrical air-flow oscillations and continuous positive pressure above baseline or PEP. These findings are consistent with the operational characteristics described by the manufacturer. ${ }^{10}$ Depending on the setting, the manufacturer reports the device will produce either a continuous positive pressure above baseline or a pressure increase from zero to a maximum level with a drop back to zero. ${ }^{9}$

It is important to note that all devices produced asymmetrical waveforms as seen in Figure 5. For the Acapella, Aerobika, and Flutter, when oscillations could be visually detected, there was a crescendo effect. Pressure amplitude was the least during the start of exhalation and peaked at mid-exhalation. Pressure amplitude gradually decreased as expiratory flow decreased nearing the end of exhalation. However, for the RC-Cornet, very little crescendo effect or pressure amplitude was observed.

The Aerobika was the only device that provided the most consistent pressure amplitude for air-flow oscillations across the spectrum of resistance settings. At the medium and high resistance settings, the Aerobika produced the highest mean pressure amplitude. At the medium and low settings, the RC-Cornet produced a mean pressure amplitude of $1 \mathrm{~cm} \mathrm{H}_{2} \mathrm{O}$. This minimal difference 


\section{Evaluation of 4 Oscillatory PEP Devices}

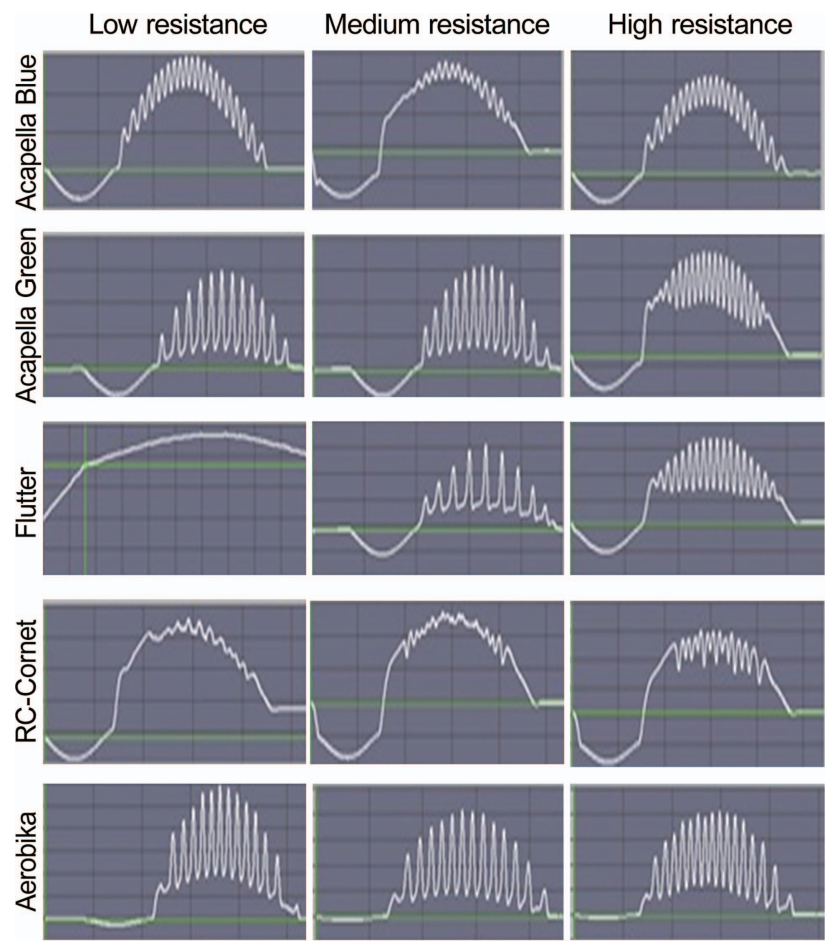

Fig. 5. Representative waveforms for each of the devices tested from which the oscillatory $f$ was counted. The $x$ axis represents the total cycle time of $2.7 \mathrm{~s}$. Each representative waveform begins with inspiration (lasting $0.6 \mathrm{~s}$ ). The oscillations occur during the active exhalation, which lasts $1.7 \mathrm{~s}$. The y axis represents peak pressure, measured in $\mathrm{cm} \mathrm{H}_{2} \mathrm{O}$. The ASL 5000 automatically adjusts the pressure scale range, resulting in differently scaled y axes for each of the graphs. The height of the $y$ axes varies in magnitude from a minimum of $4 \mathrm{~cm} \mathrm{H}_{2} \mathrm{O}$ to a maximum of $25 \mathrm{~cm} \mathrm{H}_{2} \mathrm{O}$. The green lines denote the reference $0 \mathrm{~cm} \mathrm{H}_{2} \mathrm{O}$.

in Ppeak and PEP made it difficult to visually distinguish individual air-flow oscillations, which may have resulted in reporting a fewer oscillations for these resistance settings compared with those produced at the high resistance setting.

In animal models and a small sample of subjects with bronchiectasis, Freitag et $\mathrm{al}^{11}$ demonstrated the effect turbulent spikes had on mucociliary movement. Turbulent spikes created by high frequency air-flow oscillations, approximately $15 \mathrm{~Hz}$, increased drag on the mucus on the airway walls, which resulted in increased cephalad mucus movement. The pressure amplitude reflects the turbulent spike intensity or the pressure build-up during the occlusion. A higher pressure build-up during occlusion would result in a greater burst of expiratory flow burst, thereby enhancing the mucocillary transport effect. ${ }^{11}$

The pressure output of the Flutter (Ppeak, PEP, and pressure amplitude) varied with resistance setting. Our simulated results for the Flutter were similar to findings by Alves et al, ${ }^{12}$ who reported an obvious influence of position (upward and downward tilting of the device) and flow through the device on its pressure output. Variations in the Ppeak, PEP, and pressure amplitude with the blue and green Acapella devices, at the high and low resistance settings, may be due to the operational specifications of the devices. Acapella green is indicated for patients who can sustain $\geq 3$ s of expiratory flow $\leq 15 \mathrm{~L} / \mathrm{min}$, and Acapella blue is indicated for patients with expiratory flow $\geq 15 \mathrm{~L} / \mathrm{min} .{ }^{10}$ Our model exceeded the operational specifications of these devices, at the extremes (low for the Acapella green and high for the Acapella blue). Our findings with the Flutter and Acapella devices were similar to those reported by Volsko et al $^{13}$ using continuous flow rather than an active exhalation through the device.

Our study results demonstrated a significant interaction effect of time by device type by set resistance level. These results build upon those previously reported by Volsko et $a l,{ }^{13}$ leading us to the conclusion that the tested OPEP devices produced a pressure waveform output that consistently varied. This relationship between expiratory flow, pressure waveform, and amplitude creates short bursts of high flow that result when pressure build-up behind the occlusion is released. Our results demonstrate that the resistance setting and device design, specifically the manner in which the occlusion is created, affect pressure build-up, which manifests as Ppeak when active exhalation begins and PEP when flow decays. The interaction between the variables of interest and time are important because as these disposable devices are used repeatedly as part of an airway clearance regimen, the efficacy of secretion removal may be affected by the OPEP device's ability to deliver pressures sufficient to create short flow bursts.

Clinical studies assessing the efficacy of mucociliary clearance in subjects with cystic fibrosis report higher flow bursts, and oscillations with a greater amplitude resulted in increased secretion expectoration. ${ }^{14,15}$ A reduction in the viscoelastic properties of mucus was noted with flow bursts that were transmitted as high spikes of turbulence or air flow pulsations to the upper and lower airways, which also facilitated the cephalad movement and expectoration of airway secretions. ${ }^{3}$ The therapeutic effectiveness of the air-flow oscillations is in part dependent on the ability of the device to generate and maintain a pressure amplitude or turbulent spike throughout the maneuver. ${ }^{3,14,15}$

In the clinical setting, efficacy of the OPEP therapy may be affected by device selection. Should a practitioner need to choose between the green and blue Acapella, for instance, knowledge of the patient's expiratory flows may be helpful when initially selecting the device (green or blue). The efficacy of the therapy may be affected by changes in a patient's expiratory flow during the course of therapy. For hospitalized patients, improvements in lung function may exceed the operational specifications of the Acapella used. Conversely, the efficacy of OPEP therapy performed as a routine home care regimen may be adversely affected 


\section{Evaluation of 4 Oscillatory PEP Devices}

by a decline in lung function secondary to an exacerbation or disease progression.

The literature offers a dearth of high-quality data for resistance setting selection to produce optimal frequencies, pressures that will enhance mucous clearance. Therefore, it is imperative for respiratory therapists to use their clinical assessment skills and thoroughly evaluate the patient's response to therapy on a continual basis and adjust the resistance setting and/or device used as the patient's expiratory flow, airway resistance, and degree of airway obstruction change.

There were limitations to this study. The evaluation was conducted using a lung simulator, programmed to model the pulmonary characteristics of a patient requiring OPEP therapy. This simulation was unable to account for variations in effort or airway resistance that would naturally occur clinically before, during, and after an OPEP maneuver. The researchers were unable to mimic the changes in airway resistance that occurs clinically as secretions are loosened and mobilized during OPEP and with a cough.

When used clinically, the patient's peak expiratory flow may affect the manner in which the OPEP device may produce Ppeak, pressure amplitude, oscillatory f, and PEP. Peak expiratory flow may vary with time (repeated exhalations through the device during a therapy session) due to a variety of factors, including patient effort, pain, and the presence of airway secretions. However, in this simulated model, all breaths were performed with equal effort, and the peak expiratory flow did not vary, and this model was unable to show the effect that variances in peak expiratory flow may have on the variables of interest. Additionally, the characteristics of the model were limited to only one clinical condition.

\section{Conclusions}

Statistically significant and clinically relevant variations in PEP, Ppeak, and pressure amplitude were noted between the Acapella green, Acapella blue, Aerobika, RCCornet, and Flutter, meaning that the combination of device, time, and resistance settings affects OPEP device output for pressure amplitude and oscillatory frequency. When used clinically, there is no mechanism to monitor the pressures delivered to the patient. It is important for clinicians to recognize that although each device tested produces PEP and air-flow oscillations, functional variations exist, which may impact therapeutic effectiveness. These variations occur not only between devices, but within a device, as the resistance setting changes. Further study is warranted to evaluate the process for adjusting the device's resistance settings to optimize therapeutic outcomes.

\section{REFERENCES}

1. Chatham K, Marshall C, Campbell IA, Prescott RJ. The Flutter VRP1 device for post-thoracotomy patients. Physiotherapy 1993;79(2): 95-98.

2. Pryor JA. Physiotherapy for airway clearance in adults. Eur Respir J 1999;14(6): 1418-1424.

3. McIlwaine M. Physiotherapy and airway clearance techniques and devices. Paediatr Respir Rev 2006;7(Suppl):S220-S222.

4. Groth S, Stafanger G, Dirksen H, Andersen JB, Falk M, Kelstrup M. Positive expiratory pressure (PEP-mask) physiotherapy improves ventilation and reduces volume of trapped gas in cystic fibrosis. Bull Eur Physiopathol Respir 1985;21(4):339-343.

5. Myers TR. Positive expiratory pressure and oscillatory positive expiratory pressure therapies. Respir Care 2007;52(10):1308-1326.

6. Hart N, Polkey MI, Clément A, Boulé M, Moxham J, Lofaso F, and Fauroux B. Changes in pulmonary mechanics with increasing disease severity in children and young adults with cystic fibrosis. Am J Respir Crit Care Med 2002;166(1):61-66.

7. Blakeman TC, Branson RD. Evaluation of 4 new generation portable ventilators. Respir Care 2013;58(2):264-272.

8. Volsko TA, Hoffman J, Conger A, Chatburn RL. The effect of targeting scheme on tidal volume delivery during volume control mechanical ventilation. Respir Care 2012;57(8):1297-304.

9. Fink JB, Mahlmeister MJ. High-frequency oscillation of the airway and chest wall. Respir Care 2002;47(7):797-807.

10. Emergency Medical Products. RC-Cornet operational characteristics. http://www.buyemp.com/product/curaplex-rc-cornet. Accessed June 7, 2016.

11. Freitag L, Kim CS, Long WM, Venegas J, Wanner A. Mobilization of mucus by airway oscillations. Acta Anaesthesiol Scand Suppl 1989;90:93-101.

12. Alves LA, Pitta F, Brunetto AF. Performance analysis of the Flutter VRP1 under different flows and angles. Respir Care 2008;53(3):316323.

13. Volsko TA, DiFiore J,. Chatburn RL. Performance comparison of two oscillating positive expiratory pressure devices: Acapella versus Flutter. Respir Care 2003;48(2):124-130.

14. Konstan MW, Stern RC, Doershuk CF. Efficacy of the Flutter device for airway mucus clearance in patients with cystic fibrosis. J Pediatr 1994;124(5 Pt 1):689-693.

15. App EM, Kieselmann R, Reinhardt D, Lindemann H, Dasgupta B, King M, Brand P. Sputum rheology changes in cystic fibrosis lung disease following two different types of physiotherapy: flutter vs autogenic drainage. Chest 1998;114(1):171-177. 